\title{
Validity and Reliability of an Inertial Measurement Unit-Based 3D Angular Measurement of Shoulder Joint Motion
}

\author{
Tae-Lim Yoon \\ Department of Physical Therapy, College of Health Science, Cheongju University, Cheongju, Korea
}

Purpose: The purpose of this study was to investigate the validity and reliability of the measurement of shoulder joint motions using an inertial measurement unit (IMU).

Methods: For this study, 33 participants (32 females and 1 male) were recruited. The subjects were passively positioned with the shoulder placed at specific angles using a goniometer (shoulder flexion $0^{\circ}-170^{\circ}$, abduction $0^{\circ}-170^{\circ}$, external rotation $0^{\circ}-90^{\circ}$, and internal rotation $0^{\circ}-60^{\circ}$ angles). Kinematic data on the shoulder joints were simultaneously obtained using IMU three-dimensional (3D) angular measurement (MyoMotion) and photographic measurement. Test-retest reliability and concurrent validity were examined.

Results: The MyoMotion system provided good to very good relative reliability with small standard error of measurement (SEM) and minimal detectable change (MDC) values from all three planes. It also presented acceptable validity, except for some of shoulder flexion, shoulder external rotation, and shoulder abduction. There was a trend for the shoulder joint measurements to be underestimated using the IMU 3D angular measurement system compared to the goniometer and photo methods in all planes.

Conclusion: The IMU 3D angular measurement provided a reliable measurement and presented acceptable validity. However, it showed relatively low accuracy in some shoulder positions. Therefore, using the MyoMotion measurement system to assess shoulder joint angles would be recommended only with careful consideration and supervision in all situations.

Keywords: Photography, Range of motion, Validation studies

\section{INTRODUCTION}

The assessment of range of motion (ROM) is a fundamental part of musculoskeletal examination. ${ }^{1}$ Measurement of ROM plays a vital role in diagnosing disease, assessing disability levels, and determining treatment outcomes. ${ }^{2}$ Because the shoulder joint has the greatest ROM of all body joints, it's motion would essentially affect the ability to perform activities of daily life such as dressing, washing, and reaching above shoulder level. ${ }^{2,3}$ Therefore, evaluation of the shoulder joint ROM is essential in individuals with shoulder dysfunction.

Several instruments for measuring shoulder joint angles have been used throughout the medical profession. The most common method of measuring joint angles is a mechanical goniometer. ${ }^{4}$ Physical therapists have been trained to use this instrument since the 1980s and still use it most frequently in clinics. ${ }^{4}$ Also, photo-

Received Jun 8, 2017 Revised Jun 15, 2017

Accepted Jun 16, 2017

Corresponding author Tae-Lim Yoon

E-mail free0829@gmail.com graphs of body posture, with the help of an image-processing tool, can be used to precisely define the shoulder posture and its angles. ${ }^{5}$ With advanced technology, optoelectronic and electromagnetic measurement systems have been developed to assess the shoulder joint in 3-dimensional (3D) kinematics. ${ }^{6}$ These measurement systems have successfully presented clinical usefulness with various validity and reliability studies on each method of measurement.

Recently, inertial measurement units (IMU), inertial sensors with magnetic measurement systems, have shown great potential in estimating shoulder joint angles in 3D kinematics while using a mechanical goniometer and photographs only assesse the shoulder joint one or two dimension. Several previous studies with using IMU measurement have successfully assessed joint angles in the thorax, shoulder griddle, and lower limbs during motion tasks, mostly using small sample sizes. ${ }^{6-9}$ However, the validity and reli-

Copylight (C2017 The Korea Society of Physical Therapy

This is an Open Access article distribute under the terms of the Creative Commons Attribution Non-commercial License (Http:// creativecommons.org/license/by-nc/4.o.) which permits unrestricted non-commercial use, distribution, and reproduction in any medium, provided the original work is properly cited. 
ability of IMU for 3D angular measurement of shoulder joints have been insufficiently studied in clinical environments and statistically underpowered due to small sample sizes.

Therefore, the purpose of this study was to investigate the validity and reliability of IMU measurements of shoulder joint motion. Our hypothesis was that IMU measurements of shoulder joint motion would present good reliability and validity in a clinical environment.

\section{METHODS}

\section{Subjects}

Participants $(\mathrm{n}=33,32$ females and 1 male; mean age, $21.9 \pm 2.1$ years; mean height, $162.0 \pm 6.0 \mathrm{~cm}$; mean weight, $55.8 \pm 9.0 \mathrm{~kg}$; mean BMI, $21.2 \pm 2.4 \mathrm{~kg} / \mathrm{m}^{2}$ ) were randomly recruited from a university in Cheoungju city. Exclusion criteria were any neurological or musculoskeletal disorder, previous upper extremity surgery, recent musculoskeletal trauma, and obesity $\left(\mathrm{BMI}>25 \mathrm{~kg} / \mathrm{m}^{2}\right)$. This study was approved through the Cheongju university institutional review board for human subject research. All participants agreed to participate in the study and provided written informed consent.

\section{Experimental methods}

\section{1) Photographic measurement}

A webcam was installed on a tripod at a 3-meter distance from each subject and aligned to the shoulder joint, so it was only possible to record the picture when the webcam was perpendicular to the ground. In order to control for potential errors introduced by subjects' positioning, participants were seated on a mark placed on the floor. The images for photographic measurement were captured concurrently with the IMU-based 3D angular data collection during shoulder flexion, abduction, and external rotation at each specific angle. ${ }^{10}$ The captured image was processed using the ImageJ program (version 1.43, National Institutes of Health, Bethesda, Maryland) which can measure the joint angle, in degrees, between two lines.

The active movements of shoulder flexion, abduction, and rotation were calculated by identified landmarks (midline of trunk spine, lateral epicondyle of the humerus, central axis of shoulder rotation, olecranon process of the ulna, and styloid process of the ulna, and a plumb line in side of the subject). The flexion angle was calculated by the intersection of a line that ran through the lateral epicondyle and the central axis of the shoulder rotation and another line that ran through the central axis of the shoulder rotation and a plumb line along the subject's side. The abduction angle was formed by the intersection of a line that ran through the lateral epicondyle and the central axis of the shoulder rotation and another line that ran through the midline of the trunk spine. The external rotation angle was formed by joining the ulnar styloid process, the olecranon process of the ulna, and a vertical line that passed through the olecranon process of the ulna. ${ }^{3,11}$ The photogrammetric method showed relatively good reliability with low inconsistency (flexion, intraclass correlation coefficient $($ ICC $)=0.73$, standard error of the measure (SEM) 23; abduction, ICC $=0.73$, SEM 23; external rotation, $\mathrm{ICC}=0.62, \mathrm{SEM} 15^{\circ}$ ).

\section{2) MyoMotion 3D motion analysis system (IMU 3D angular measurement)}

The MyoMotion (Noraxon Inc., Scottsdale, AZ, USA) 3D motion analysis system was used to investigate the kinematic variables in the shoulder joint. A small IMU sensor placed on a body segment tracks its 3D angular orientation. The IMU sensors, attached with special fixation straps and elastic straps on two adjacent body segments, can calculate the joint ROM between these segments. The MyoMotion IMU sensors transmit the motion of the human body to the MyoMotion receiver to compute the angular changes of the selected joint. The MyoMotion IMU sensors include 3D accelerometer, gyroscope, and magnetometer that measure 3D rotation angles of each IMU sensor in absolute space. The Noraxon MyoMotion $3 \mathrm{D}$ motion analysis system is completely wireless and does not require the calibration of the measurement space. ${ }^{12}$ The MyoMotion IMU sensors can be placed with 16 joint segments according to the body model, which is suggested in the MR3 software (Noraxon Inc., Scottsdale, AZ, USA).

For shoulder joint assessment, 3 IMU sensors were attached to the upper arm (side attachment to the middle of the humerus), C7, and bony area of the sacrum. IMU sensor calibration for the body position was performed before every measurement. The seated position with the arm vertically aligned with the plumb line was used to determine the value of the $0^{\circ}$ angle in the shoulder joint as the calibration posture. Immediate shoulder angle changes were recorded with the sampling frequency at $200 \mathrm{~Hz}$. Positive values of the angle depending on the joint and axis corresponded to flexion, 

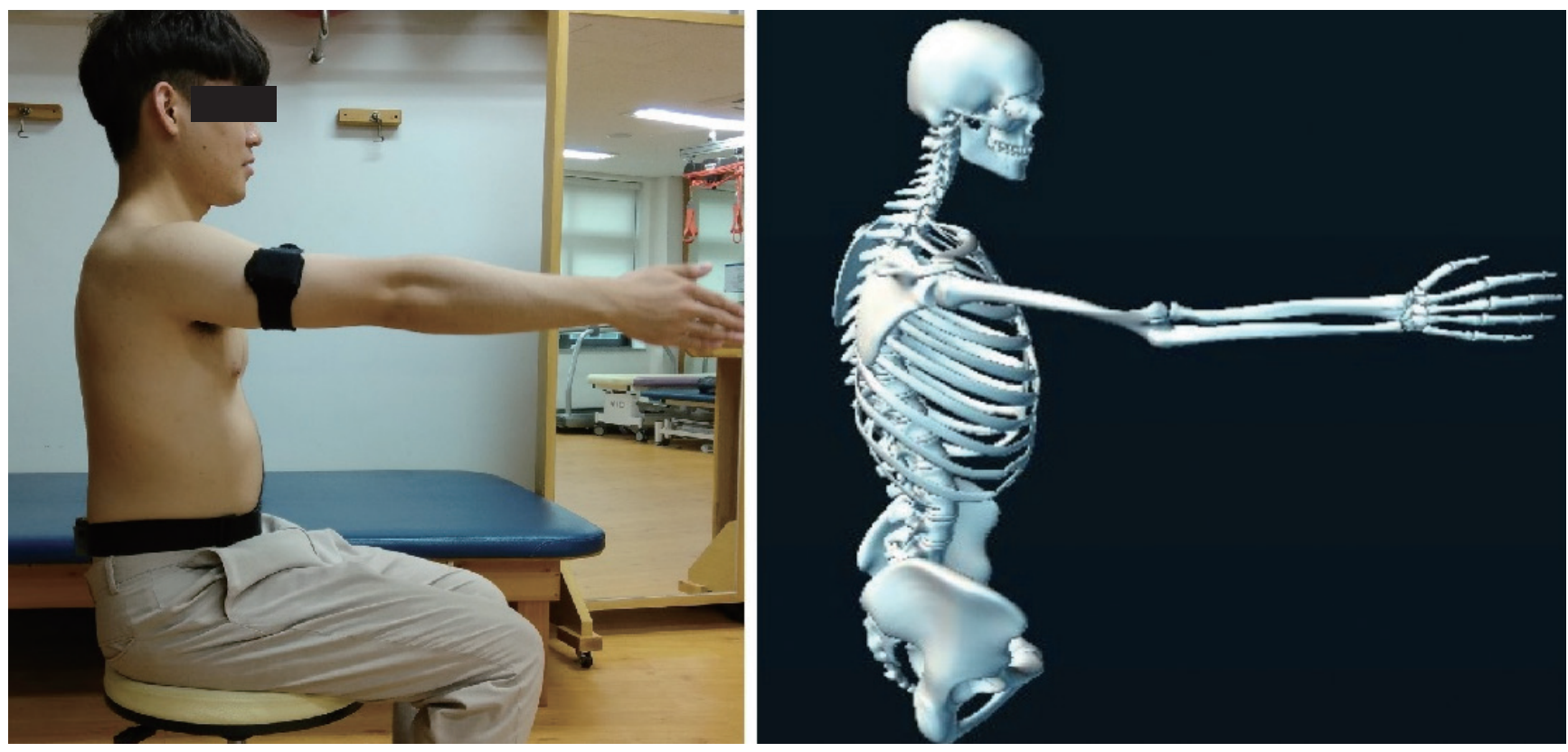

Figure 1. Inertial measurement unit-based 3D angular measurement of shoulder joint motion (MyoMotion).

abduction, and external rotation at each plane (Figure 1).

\section{3) Procedure}

A primary investigator had a practice session with the subjects for 5 min. to achieve the proper shoulder joint motions. Shoulder joint motions were measured on seated volunteer subjects to minimize the compensation movements. For shoulder flexion, the subjects were seated with the elbow extended, the wrist in a neutral position, and the palm of the hand toward the midline at the beginning and end of the movement. For shoulder abduction, the subjects were initially positioned in the same manner as for the shoulder flexion. In over-the-shoulder abduction of $90^{\circ}$, shoulder external rotation was allowed to avoid shoulder impingement. For external rotation, the subjects were seated with $90^{\circ}$ shoulder joint abduction and $90^{\circ} \mathrm{el}-$ bow flexion. The subjects were passively positioned with extremities at specific angles, which were confirmed with a goniometer (shoulder flexion $0^{\circ}, 45^{\circ}, 90^{\circ}, 135^{\circ}$, and $170^{\circ}$; abduction $0^{\circ}, 45^{\circ}, 90^{\circ}, 135^{\circ}$, and $170^{\circ}$; external rotation $0^{\circ}, 45^{\circ}$, and $90^{\circ}$; internal rotation $45^{\circ}$ and $60^{\circ}$ ). The maximum angle at each plane was determined in a pilot study to exclude compensation movements associated with shoulder joint motions.

Kinematic data on shoulder joints were simultaneously obtained using photographic measurement and the MyoMotion 3D motion analysis system during shoulder flexion, abduction, and rotation in random order. The primary investigator took a photograph of each position at specific angles from recorded video. All the photographic measurements were synchronized with the MyoMotion 3D motion analysis system. This measurement was repeated 2 times to use the averaged data to reduce measurement errors. After $10 \mathrm{~min}$., a second measurement session for shoulder joint motion was done to calculate test-retest reliability. All the data were averaged and used for statistical analysis. Therefore, a direct comparison could be completed between the IMU and the goniometer measurement and between the IMU and the photographic measurement in individual subjects.

\section{4) Statistical analysis \\ (1) Test-retest reliability}

The intra-class correlation coefficient $\operatorname{ICC}(3,2)$ was used to determine the relative test-retest reliability of an IMU 3D angular measurement of shoulder angles. ${ }^{13}$ Three ICC $(3,2)$ values, representing the agreement of two trials for each motion from the shoulder flexion, abduction, and rotation, were computed. The ICC $(3,2)$ values were defined as "poor" when below 0.20, "fair" for values of 0.21 0.40, "moderate" for values of 0.41-0.60, "good" for values of 0.610.80 , and "very good" for values of 0.81-1.0. ${ }^{14}$ The standard error of measurement (SEM) and the minimal detectable change (MDC) were calculated to find absolute reliability. The SEM was defined as 
standard deviation (SD) multiplied by the square root of the ICC subtracted from $1 .{ }^{15}$ The MDC was calculated by multiplying the SEM by the square root of $2{ }^{16}$

\section{(2) Concurrent validity}

The 95\% limits of agreement (LOA) between the IMU 3D angular measurement and the two measurement standards for the shoulder were computed for each motion to determine validity.16 To obtain the 95\% LOA in each posture, the mean of the two shoulder angle measurements from each method were calculated. Next, the mean and SD of differences between the IMU 3D angular measurements and the goniometer measurements, and between the IMU 3D angular measurements and the photographic measurements, were computed. The 95\% LOA were defined as the mean difference \pm 1.96 SD of the difference, so that $95 \%$ of differences ranged within these limits. If the $95 \% \mathrm{LOA}$ were greater than $\pm 5^{\circ}$, the differences between measurement methods were considered to be clinically significant. ${ }^{17}$ All statistical analyses were calculated by using SPSS Statistics software 23 (IBM Corp., Armonk, NY, USA).

\section{RESULTS}

\section{Test-retest reliability}

Test-retest reliability of the MyoMotion system for the measurement of shoulder joint angles with ICC, SEM, and MDC are de- scribed in Table 1. From the sagittal plane, the MyoMotion system had good to very good relative reliability for the measurement of shoulder joint flexion with ICC $(3,2)$ values. From the frontal plane, the MyoMotion system had good to very good relative reliability for the measurement of shoulder joint abduction to $0^{\circ}, 90^{\circ}$, and $170^{\circ}$ with ICC $(3,2)$ values. However, the MyoMotion system showed moderate relative reliability for abduction to $45^{\circ}$ and $135^{\circ}$. For the transverse plane, the MyoMotion system had good to very good relative reliability for the measurement of shoulder joint rotation with ICC $(3,2)$ values. Relatively small SEM and MDC values were presented for all shoulder positions from the all three planes. This result indicates good absolute reliability.

\section{Concurrent validity}

The 95\% LOA between the MyoMotion system and the goniometer and between the MyoMotion system and the photo method are presented in Table 2 . Shoulder flexion $0^{\circ}$ to $45^{\circ}$, abduction $0^{\circ}$, internal rotation $45^{\circ}$ in the MyoMotion system measurements were accurate (the 95\% LOA for the discrepancy between the measurements were within $\pm 5^{\circ}$ ) compared with the goniometer or the photo method. ${ }^{18}$ Also, shoulder flexion $90^{\circ}$ to $135^{\circ}$, rotation $0^{\circ}$ to external rotation $45^{\circ}$, and internal rotation $45^{\circ}$ to $90^{\circ}$ in the MyoMotion measurements were not accurate (the 95\% LOA for the discrepancy between the measurements exceeded $\pm 5^{\circ}$ ) compared with the goniometer or the photo method. However, shoulder abduction $45^{\circ}$ to

Table 1. Test-retest reliability results of the MyoMotion for the measurement of shoulder angle

\begin{tabular}{|c|c|c|c|c|c|}
\hline & Position & ICC & Mean & SEM & MDC \\
\hline \multirow[t]{5}{*}{ Sagittal Plane } & Flexion $0^{\circ}$ & 0.81 & -0.7 & 0.58 & 0.82 \\
\hline & Flexion $45^{\circ}$ & 0.71 & 38.5 & 1.23 & 1.74 \\
\hline & Flexion $90^{\circ}$ & 0.72 & 77.6 & 1.40 & 1.97 \\
\hline & Flexion $135^{\circ}$ & 0.68 & 124.1 & 1.55 & 2.19 \\
\hline & Flexion $170^{\circ}$ & 0.98 & 145.7 & 2.00 & 2.83 \\
\hline \multirow[t]{5}{*}{ Frontal Plane } & Abduction $0^{\circ}$ & 0.82 & 3.7 & 0.83 & 1.17 \\
\hline & Abduction $45^{\circ}$ & 0.51 & 33.7 & 1.49 & 2.11 \\
\hline & Abduction $90^{\circ}$ & 0.65 & 74.2 & 1.42 & 2.00 \\
\hline & Abduction $135^{\circ}$ & 0.43 & 121.3 & 1.48 & 2.09 \\
\hline & Abduction Max $170^{\circ}$ & 0.93 & 147.7 & 1.87 & 2.64 \\
\hline \multirow[t]{5}{*}{ Transverse Plane } & ROT $0^{\circ}$ & 0.89 & -4.9 & 1.80 & 2.55 \\
\hline & External ROT $45^{\circ}$ & 0.74 & 41.4 & 2.59 & 3.66 \\
\hline & External ROT Max $90^{\circ}$ & 0.79 & 77.5 & 3.56 & 5.03 \\
\hline & Internal ROT $45^{\circ}$ & 0.83 & 46.9 & 3.24 & 4.58 \\
\hline & Internal ROT Max $60^{\circ}$ & 0.75 & 52.4 & 3.16 & 4.47 \\
\hline
\end{tabular}

ICC: intra class correlation coefficient, MDC: minimal detectable change, ROT: rotation, SEM: standard error of the measure. 
Table 2. 95\% limits of agreement between measurements of shoulder joint angle obtained using the IMU, goniometer, and photo methods

\begin{tabular}{|c|c|c|c|c|c|}
\hline & \multirow{2}{*}{ Position } & \multicolumn{2}{|c|}{ MyoMotion VS Goniometer } & \multicolumn{2}{|c|}{ MyoMotion VS Photo } \\
\hline & & Mean bias & 95\% Limits of agreement & Mean bias & 95\% Limits of agreement \\
\hline \multirow[t]{5}{*}{ Sagittal Plane } & Flexion $0^{\circ}$ & -0.4 & 0.00 to -0.94 & -4.7 & -3.72 to -5.98 \\
\hline & Flexion $45^{\circ}$ & -2.3 & -1.35 to -3.26 & -3.4 & -1.28 to -5.42 \\
\hline & Flexion $90^{\circ}$ & -5.6 & -4.38 to -6.93 & -8.9 & -6.58 to -11.3 \\
\hline & Flexion $135^{\circ}$ & -4.9 & -3.67 to -6.14 & -6.2 & -3.90 to -8.48 \\
\hline & Flexion $170^{\circ}$ & -11.8 & -10.38 to -13.21 & -20.4 & -18.16 to -22.76 \\
\hline \multirow[t]{5}{*}{ Frontal Plane } & Abduction $0^{\circ}$ & 4.1 & 2.86 to 5.31 & 0.1 & -1.15 to 1.49 \\
\hline & Abduction $45^{\circ}$ & -11.3 & -13.79 to -8.77 & -12.1 & -14.54 to -9.56 \\
\hline & Abduction $90^{\circ}$ & -17.4 & -20.40 to -14.47 & -13.9 & -16.96 to -10.98 \\
\hline & Abduction $135^{\circ}$ & -17.9 & -22.14 to -13.65 & -22.6 & -26.82 to -18.48 \\
\hline & Abduction $170^{\circ}$ & -25.1 & -28.29 to -21.99 & -27.9 & -31.27 to -24.68 \\
\hline \multirow[t]{5}{*}{ Transverse Plane } & ROT $0^{\circ}$ & -5.1 & -7.41 to -2.82 & -7.9 & -10.57 to -5.34 \\
\hline & External ROT $45^{\circ}$ & -7.2 & -11.02 to -3.41 & -9.1 & -12.82 to -5.41 \\
\hline & External ROT $90^{\circ}$ & -17.0 & -22.53 to -11.52 & -14.9 & -20.30 to -9.51 \\
\hline & Internal ROT $45^{\circ}$ & -5.0 & -5.04 to 4.90 & -4.8 & -9.57 to -0.04 \\
\hline & Internal ROT $60^{\circ}$ & -7.6 & -2.90 to 12.20 & 6.9 & 2.05 to 11.86 \\
\hline
\end{tabular}

ROT: rotation.

$135^{\circ}$ presented significantly different angles (the 95\% LOA for the discrepancy between systems exceeded $\pm 10^{\circ}$ ). In particular, shoulder abduction $170^{\circ}$ in the MyoMotion system measurement showed the largest difference. There was a trend for shoulder joint measurements to be underestimated using IMU-based 3D angular measurement system compared to the goniometer and photo methods in all planes.

\section{DISCUSSION}

The purpose of this study was to investigate the validity and reliability of IMU measurements of shoulder joint motions in a clinical environment. Although IMU 3D measurements have shown great potential for estimating body posture in 3D kinematics, the validity and reliability of IMU for 3D angular measurement of shoulder joint motions had not been fully examined in the clinical environment at the time of this study.

The MyoMotion system had good to very good relative reliability for the measurement of all shoulder positions except the shoulder abduction $45^{\circ}-135^{\circ}$, which showed only moderate test-retest reliability. We expected that the uncontrolled shoulder motion in 3D during the shoulder abduction would influence the results. Several previous studies that measured joint angles using the inertial sensors showed good to excellent test-retest reliability, similar to our study. ${ }^{19,20}$
These studies claimed that the motion of the upper body showed excellent ICC values with relatively small SEM and MDC values from the all three planes. These results indicate good relative and absolute reliability. Similar to our study, a previous study reported relatively small errors from $2.3^{\circ}$ to $4.8^{\circ}$ in upper limb movement. ${ }^{21}$ Usually, measurement errors of between $2^{\circ}$ to $5^{\circ}$ are also likely to be regarded as reasonable. ${ }^{22}$ However, this other study insisted that the degree of reliability depended on location and was task specific, although the IMU sensors were considered a reliable method. ${ }^{22}$ Therefore, using the MyoMotion system to measure the shoulder joint angle is recommended with careful consideration and supervision.

The 95\% LOA between the IMU 3D angular measurement and two other measurement standards for the shoulder joint angles were computed to determine the concurrent validity of the MyoMotion system. From the sagittal plane, the MyoMotion system had relatively good results for shoulder flexion $0^{\circ}$ to $135^{\circ}$. A study insisted that the 95\% LOA for the discrepancy between the measurements were within $\pm 5^{\circ}$ and would have a good validity. 18 Also, for other $3 \mathrm{D}$ motion analysis systems, approximately $12^{\circ}$ to $20^{\circ}$ maximum error has been reported in the more advanced motion systems. ${ }^{23,24}$ Therefore, using the MyoMotion system to assess shoulder flexion $0^{\circ}$ to $135^{\circ}$ seems to fall within an acceptable validity range. However, the MyoMotion system presented significantly increased mean 
bias for shoulder flexion $170^{\circ}$ to as much as $-11.8^{\circ}$ (versus the goniometer) and $-20.4^{\circ}$ (versus the photo method). The mean bias for shoulder flexion $170^{\circ}$ may be large enough to misinform clinical interpretation. Consequently, using the MyoMotion system to assess for shoulder flexion over $135^{\circ}$ should be avoided.

From the frontal plane, the MyoMotion system had poor results for the measurement of shoulder abduction except in the starting position. Shoulder abduction $45^{\circ}$ to $135^{\circ}$ presented significantly different angles $\left(-11.3^{\circ}\right.$ to $\left.-17.4^{\circ}\right)$ compared to goniometer and photo methods. In particular, shoulder abduction $170^{\circ}$ in the MyoMotion system measurements showed the largest mean bias $\left(25.1^{\circ}\right)$. This unexpectedly large mean bias could have been caused by the complicated 3D shoulder joint motions. During the shoulder abduction measurement in high degrees, an external rotation of the shoulder was performed to clear the acromion process. ${ }^{25}$ We assumed that the uncontrolled rotation in the shoulder joint during the shoulder abduction affected the results for the measurement of the shoulder abduction. Therefore, more study of shoulder abduction in the frontal plane with control of rotational movement of the shoulder joint is required.

From the transverse plane, the MyoMotion system had slightly different angles for the measurement of shoulder rotation except external rotation $90^{\circ}$. The mean bias $\left(0^{\circ}\right.$ to $\left.-7.6^{\circ}\right)$ in the shoulder rotation at external rotation $45^{\circ}$ to internal rotation $60^{\circ}$ showed a relatively acceptable range. However, the result in shoulder external rotation $90^{\circ}$ reported unacceptable validity. We therefore recommend using the MyoMotion system to assess external shoulder rotation $45^{\circ}$ to internal rotation $60^{\circ}$ only.

Finally, there was a trend for shoulder joint measurements to be underestimated using the MyoMotion system compared to the goniometer and photo methods in all planes. This result indicates the systematic bias in our study. The most likely reason would be the calibration process before starting the measurements. We used the calibration posture of sitting with the elbow extended. However, the calibration posture was relatively inconstant and affected by each subject's body shape. Therefore, we suggest conducting a calibration testing session for accuracy before measuring the shoulder angles.

Our study has some limitations. First of all, the goniometer and photo methods were 2D measurement systems. Therefore, comparing 2D and 3D measurement systems has resulted in some differences. Second, our data has a limitation in generalization to male because the subjects in this study were mostly women. Also, the compensation movements of other body parts, such as the scapula and trunk, were not fully controlled, although a skilled primary investigator observed these problems while supervising the measurements. Therefore, we suggest that further validity studies between IMU 3D measurement and other 3D measurement systems are required.

\section{ACKNOWLEDGEMENTS}

This work was supported by the research grant of Cheongju University in 2015 .

\section{REFERENCES}

1. Awan R, Smith J, Boon AJ. Measuring shoulder internal rotation range of motion: a comparison of 3 techniques. Arch Phys Med Rehabil. 2002; 83(9):1229-34.

2. Terwee CB, de Winter AF, Scholten RJ et al. Interobserver reproducibility of the visual estimation of range of motion of the shoulder. Arch Phys Med Rehabil. 2005;86(7):1356-61.

3. Hayes K, Walton JR, Szomor ZR et al. Reliability of five methods for assessing shoulder range of motion. Aust J Physiother. 2001;47(4):289-94.

4. Williams JG, Callaghan M. Comparison of visual estimation and goniometry in determination of a shoulder joint angle. Physiotherapy. 1990; 76(10):655-7.

5. Omkar SN, Kumar MM, Mudigere D. Postural assessment of arbitrarily taken portrait and profile photographs using ImageJ. J Bodyw Mov Ther. 2007;11(3):231-7.

6. Cutti AG, Giovanardi A, Rocchi L et al. Ambulatory measurement of shoulder and elbow kinematics through inertial and magnetic sensors. Med Biol Eng Comput. 2008;46(2):169-78.

7. de Vries WH, Veeger HE, Cutti AG et al. Functionally interpretable local coordinate systems for the upper extremity using inertial \& magnetic measurement systems. J Biomech. 2010;43(10):1983-8.

8. Faber GS, Kingma I, Bruijn SM et al. Optimal inertial sensor location for ambulatory measurement of trunk inclination. J Biomech. 2009;42(14): 2406-9.

9. Kang GE, Gross MM. Concurrent validation of magnetic and inertial measurement units in estimating upper body posture during gait. Measurement. 2016;82:240-5.

10. Maykut JN, Taylor-Haas JA, Paterno MV et al. Concurrent validity and reliability of $2 \mathrm{~d}$ kinematic analysis of frontal plane motion during running. Int J Sports Phys Ther. 2015;10(2):136-46.

11. Bullock MP, Foster NE, Wright CC. Shoulder impingement: the effect of sitting posture on shoulder pain and range of motion. Man Ther. 2005; 10(1):28-37.

12. Struzik A, Konieczny G, Stawarz M et al. Relationship between lower limb angular kinematic variables and the effectiveness of sprinting during the acceleration phase. Appl Bionics Biomech. 2016;2016:7840709. 
13. Shrout PE, Fleiss JL. Intraclass correlations: uses in assessing rater reliability. Psychol Bull. 1979;86(2):420-8.

14. Landis JR, Koch GG. The measurement of observer agreement for categorical data. Biometrics. 1997;33(1):159-74.

15. Stratford PW, Binkley JM, Riddle DL. Health status measures: strategies and analytic methods for assessing change scores. Phys Ther. 1996; 76(10):1109-23.

16. Stratford PW, Binkley J, Solomon P et al. Defining the minimum level of detectable change for the Roland-Morris questionnaire. Phys Ther. 1996;76(4):359-65.

17. Bland JM, Altman DG. Statistical methods for assessing agreement between two methods of clinical measurement. Lancet. 1986;8(1):307-10

18. Huber ME, Seitz AL, Leeser M et al. Validity and reliability of Kinect skeleton for measuring shoulder joint angles: a feasibility study. Physiotherapy. 2015;101(4):389-93.

19. Leardini A, Lullini G, Giannini $S$ et al. Validation of the angular measurements of a new inertial-measurement-unit based rehabilitation system: comparison with state-of-the-art gait analysis. J Neuroeng Rehabil.
2014;11:136.

20. Orlowski K, Eckardt F, Herold F et al. Examination of the reliability of an inertial sensor-based gait analysis system. Biomed Tech (Berl). 2017.

21. Zhou H, Stone T, Hu H et al. Use of multiple wearable inertial sensors in upper limb motion tracking. Med Eng Phys. 2008;30(1):123-33.

22. Cuesta-Vargas AI, Galán-Mercant A, Williams JM. The use of inertial sensors system for human motion analysis. Phys Ther Rev. 2010;15(6): 462-73.

23. Gribble P, Hertel J, Denegar C et al. Reliability and validity of a 2-D video digitizing system during a static and a dynamic task. J Sport Rehabil. 2005;14(2):137-49.

24. Richards JG. The measurement of human motion: a comparison of commercially available systems. Hum Mov Sci. 1999;18(5):589-602.

25. Reinold MM, Wilk KE, Fleisig GS et al. Electromyographic analysis of the rotator cuff and deltoid musculature during common shoulder external rotation exercises. J Orthop Sports Phys Ther. 2004;34(7):385394. 